\title{
Das Private dringt in die Öffentlichkeit. Automediale und biopolitische Dimensionen des Atems in der Kunst der polnischen Gruppe Werkstatt der Filmform (1970-1977)
}

\begin{abstract}
Die polnische Kunst der 1970er Jahre war oft nicht nur stark politisch geprägt. Sie spielte zudem mit dem Potenzial des Mediums und stellte mit konzeptuellem Scharfsinn die Frage nach Verfassung, Status und Definition der Kunst überhaupt. Das Motiv des Atems taucht in jenem Jahrzehnt in den Arbeiten der polnischen Neoavantgarde mehrfach prominent auf. ${ }^{1}$ Man betrachtete die Atmung als Ausdruck des lebendigen, individuellen Körpers, die einerseits in die Wirklichkeit verstrickt ist und andererseits dem medialen Kontext der Kunst angehört. Weiterhin diente der Atem als Metapher, die einen Anstoß zur Dekonstruktion der politischen Propaganda geben konnte. Der Atem kann zudem als ein Gradmesser dafür gesehen werden, auf welche strikte und zugleich komplexe Weise im Sozialismus Privatheit und Öffentlichkeit voneinander getrennt waren. Die Sphäre des Privatlebens wurde schon seit den 1960er Jahren als eine Art Freiheitszone betrachtet und zelebriert, zu der die kommunistische Partei keinen Zugang hatte und die sie nicht ganz kontrollieren konnte. In den 70er Jahren herrschte in der Volksrepublik Polen ein scharfer Dualismus: Privatheit und Öffentlichkeit wurden voneinander stark getrennt. ${ }^{2}$ Privatheit, das Al1tägliche und Körperlichkeit waren also politisch definierte Zonen, in denen man ,freier atmen" durfte.
\end{abstract}

1 Aufgrund des beschränkten Textumfangs verzichte ich hier auf die Analyse solcher Werke der polnischen Neoavantgarde wie z. B. Moment Art-Gemeinsamer Atemdruck (1976-1980) von Wincenty Duniko-Dunikowski, Träumen (1978) von Natalia LL und Der Atem (1981) von Teresa Tyszkiewicz. Duniko-Dunikowski verhalf noch dem trivialsten Moment der Existenz zu seinem Wert, indem er Atemzüge auf Glasplatten ,druckte‘ und darauf hinwies, wie wichtig die alltägliche - aus der offiziellen, pathetischen Ikonosphäre des sozialistischen Polens verdrängte - Banalität der Existenz ist. Tyszkiewicz wiederum hat in inrem Video Der Atem Stereotype dekonstruiert, die die Frau objektivieren und essentialisieren.
2 Elzbieta Tarkowska/Jacek Tarkowski, ,,Amoralny familizm czyli o dezintegracji społecznej w Polsce lat osiemdziesiątych“, in: Jacek Tarkowski (Hg.), Socjologia świata polityki. Władza i społeczeństwo w systemie autorytarnym, Warschau 1994, S. 264. Siehe auch: Stanley I. Benn, „Privacy, Freedom and Respect for Person“, in: J. Roland Pennock/John W. Chapman (Hg.), Privacy, New York 1971, S. 1-26; Bartłomiej Kamiński, The Collapse of State Socialism: The Case of Poland, Princeton 1991, Kapitel 6. 
Ziel dieses Textes ist eine Analyse der Funktionsweisen des Atems im Kunstschaffen von zwei Mitgliedern der Gruppe Werkstatt der Filmform (Warsztat Formy Filmowej, 1970-1977), einer der wichtigsten Gruppen der polnischen Neoavantgarde. Meine Analyse bezieht sich dabei sowohl auf die politische Propaganda als auch auf das Potential des Mediums Film. Łukasz Ronduda bezeichnet die 1970 an der Filmschule Lodz von Studenten und Absolventen der Studiengänge Kamera und Regie gegründete Gruppe Werkstatt der Filmform als die „wichtigste Gruppierung des strukturellen Films in Ostmitteleuropa“. Die Gründer und Mitglieder dieser rein männlichen Gruppe wie Józef Robakowski, Ryszard Waśko, Paweł Kwiek, Wojciech Bruszewski, Zbigniew Rybczyński, Janusz Połom, Antoni Mikołajczyk, Kazimierz Bendkowski und Andrzej Różycki lehnten, so Ronduda, nicht nur die „klassische Erzählweise und filmische Illusion“ ab, sondern „sogar (bei manchen Regiearbeiten) den gesamten kinematografischen ,Apparat '“. ${ }^{3}$ Inspirieren ließen sie sich vornehmlich von den bildenden Künsten, vor allem den Strömungen des Konstruktivismus und der analytischen Konzeptkunst. Paradoxerweise hatten die Mitglieder der Gruppe Werkstatt der Filmform relativ viel Freiheit, weil sie sich auf die Struktur des Mediums Film selbst und auf die Befreiung vom Illusionismus konzentrierten, ohne direkt politische Kritik zu äußern. In den 70er Jahren strebte die polnische Regierung sowohl im Ostblock als auch im Westen nach politischer Anerkennung als tolerante Macht, weswegen es vielen Kunstakademien, Kunstinstitutionen und Galerien erlaubt war, medienkritische Kunst auszustellen. ${ }^{4}$ Daher agierte die Gruppe Werkstatt der Filmform tatsächlich unabhängig von staatlichen Vertriebsmechanismen und Repertoirepolitik. Ihre Mitglieder organisierten in dieser Zeit viele alternative Veranstaltungen, ohne offizielle Juroren einzubeziehen oder auf staatliche Auszeichnungen zu zählen. Alle Veranstaltungen und Filmproduktionen, deren Kosten sie von Anfang bis Ende selbst trugen, waren nicht kommerziell und kostenfrei zugänglich. ${ }^{5}$ Die Regierung von Edward Gierek versuchte auf der internationalen Bühne Polen als ein westliches und modernes Land zu repräsentieren:

3 Siehe Lukasz Ronduda, „Warsztat Formy Filmowej. Realizacje filmowe z lat 1970-1977“, in: Artpapier.com 2004, verfügbar unter: http://artpapier.com/pliki/ archiwum_sierpien_04/recfilm/warsztatyformy.html (letzter Zugriff: 4.5.2020). Übers. aus dem Polnischen v. Hans Gregor Njemz. Wenn nicht anders angegeben, gilt dies auch für alle weiteren Übersetzungen. Siehe weiterführend Ryszard W. Kluszczyński, Warsztat Formy Filmowej, Ausst.-Kat. CSW Zamek Ujazdowski, Warschau 2000; Marika Kuźmicz/Lukasz Ronduda (Hg.), Warsztat Formy Filmowej / Workshop of the Film Form, Warschau 2017.
4 Vgl. Piotr Piotrowski, „Post-modernism and Post-totalitarianism. The Poland Case of the 1970s", in: ARS 2-3, 1993, Bratislava 1994, S. 231-242; Lechosław Olszewski, „Działalność Warsztatu Formy Filmowej jako przykład strategii sztuki wobec władzy w Polsce lat siedemdziesiątych“, in: Artium Quaestiones 9, 1998, S. 111-155.

5 Alicja Cichowicz, „Warsztat Formy Filmowej“, in: Katalog artystów z województwa łódzkiego, verfügbar unter: http://www.artysci-lodzkie.pl/pl/artysta/w/warsztatformy-filmowej (letzter Zugriff: 24.5.2020). 
„Die Behörden übernahmen einen neo-avantgardistischen [...] Diskurs.

Solche Kunst war für die Macht notwendig. Einerseits wurden von den Künstlern Neutralität und unkritische Einstellung gefordert, während andererseits die künstlerischen Aktivitäten, formalen Experimente und modernen Stilformen vom ,Okzidentalismus` der Gesellschaft zeugen sollten."6

Diese von der Gruppe Werkstatt der Filmform durchgeführten Formexperimente und automedialen Analysen wandten sich aber oft subversiv gegen die Macht, wodurch die Grenze zwischen autoanalytischer und engagierter Kunst verschwamm. ${ }^{7} 1977$ löste sich die Gruppe auf, da sich inzwischen die meisten Künstler anderen künstlerischen Strategien zugewandt hatten.

Im vorliegenden Text sollen zwei Arbeiten analysiert werden: Ich gehe (1973; Sammlung des Museum für Gegenwartskunst in Warschau) ${ }^{8}$ von Józef Robakowski und Video-Atem - der Informationskanal (1978; Sammlung der Stiftung Arton, Warschau) von Paweł Kwiek. Die Bedeutung und Konzeption des Atems wurden für beide Werke bislang keiner eingehenden Analyse unterzogen. Eine detaillierte Untersuchung im Hinblick auf dieses Motiv gestattet es, die Verfasstheit der polnischen Kunst der siebziger Jahre insgesamt zu diagnostizieren, sowohl im Kontext der Definition des Mediums und der Kunst selbst, als auch in Bezug auf die damalige gesellschaftspolitische Situation. Ein besonderes Augenmerk wird auf die künstlerische Auseinandersetzung mit Staatsmacht und Biopolitik gelegt.

Der Begriff Biopolitik wird hier in Rekurs auf Gilles Deleuze verstanden, der Michel Foucaults Überlegungen re-interpretierte. ${ }^{9}$ Deleuze interessiert sich im Kontext der Biopolitik und Biomacht unter anderem für die Kontrolle über den Körper. Das Foucaultsche Prinzip der Disziplin wurde von ihm durch den Begriff der Kontrolle ersetzt, welche durch die Schaffung vermeintlicher Freiheiten wirksam wird..$^{10}$ Während der Körper in der Volksrepublik Polen offiziell als ein Teil der perfekten Maschine zu funktionieren hatte, musste der private Körper unsichtbar bleiben und wurde so aus der Politik ausgeschlossen. Die eingeräumte Freiheit im Privatraum wurde mit dem Ausschluss aus der ÖffentlichPeschken.

7 Ebd., S. 116.

8 Video online verfügbar unter: https://www.youtube.com/ watch?v=8cC-xRLMelk\&t=3s (letzter Zugriff: 9.4.2020).

9 Für einen Überblick zu Konzepten der Biopolitik siehe: Andreas Folkers/Thomas Lemke (Hg.), Biopolitik Ein Reader, Berlin 2014.

10 Vgl. Gilles Deleuze, „Postskriptum über die Kontrollgesellschaften“ (1990), in: ders. (Hg.), Unterhandlungen 1972-1990, Frankfurt am Main 1993, S. 254-262. 
keit bezahlt. In den hier analysierten Kunstwerken fungiert der Atem in diesem Sinne als grenzüberschreitende Figur, mit der Privatheit und Öffentlichkeit des Körpers kritisch miteinander verhandelt werden. Beide Mitglieder der Werkstatt der Filmform überschreiten dabei die Grenze zwischen autoanalytischer und engagierter Kunst und lassen den Körper als Politikum sichtbar werden.

\title{
Robakowski als atemloser Antivoyeur
} Józef Robakowski (geb. 1939), Kamerastudent der Staatlichen Film-, Fernseh- und Theaterhochschule in Lodz, gehörte ab $1970 \mathrm{zu}$ den Mitorganisatoren der Avantgardegruppe Werkstatt der Filmform. Auf der Tradition des Konstruktivismus und Konzeptualismus aufbauend, analysierten die Mitglieder dieser Gruppe die Sprache der Medien, vor allem des Films und der Fotografie, und trachteten danach, sie von der Tendenz zu Narration und Illusion zu befreien. Robakowski warf damals die Frage auf, ob es eine ureigene Sprache des Mediums Film gebe. In seinem Manifest Noch einmal zum , reinen Film " hob er 1970 hervor:

\begin{abstract}
„Gegenstand meiner Arbeit ist es derzeit, den Film von Elementen zu befreien, die der literarischen Ausdrucksform eigen sind. Ich bin mir bewusst, dass dieses Konzept die Handlungsfreiheit einschränkt, künstliche Barrieren errichtet und in den Randbereich der Gattung führt. Dennoch glaube ich, ja bin sogar überzeugt, dass es mir durch verschiedenerlei Forschungen, Versuche und Vorschläge gelingen wird, den Film vom Ballast der aus der Literatur stammenden Gewohnheiten zu befreien, die sowohl von Filmschaffenden als auch Zuschauern fast einhellig kritiklos übernommen worden sind.“11
\end{abstract}

Die Strategie der Werkstatt der Filmform war demnach zweifellos politisch, subversiv und kritisch gegenüber der damaligen polnischen Filmproduktion, die an der Verfestigung eines Idealbildes vom sozialistischen Polen arbeitete. Die Lodzer Filmschule bildete schließlich auch das zukünftige Personal des Fernsehens aus - eines der Hauptwerkzeuge der damaligen staatlichen Propaganda. Jegliches filmische Experiment wurde daher als politische Stellungnahme verstanden. ${ }^{12}$ Robakowski experimentierte u. a. in einer Filmreihe mit dem Titel Mechanisch-biologische Aufzeichnungen (1971-1978) ${ }^{13}$, indem er die

11 Józef Robakowski, „Jeszcze raz o czysty film“, in: Polska 10, 1971, o. s.

12 Vgl. Łukasz Guzek, Rekonstrukcja sztuki akcji w Polsce, Warschau/Thorn 2017, S. 202.

13 Siehe Karolina Jabłońska, „Józef Robakowski“, in: Anna Saciuk-Gąsowska (Hg.), For Encouragement to Museum. Collection of the Lodz Association for the Encouragement of Fine Art, Lodz 2013, S. 132. 
Kamera vom Auge wegführte und sie, seinen Körperbewegungen folgend, mechanisch aufzeichnen ließ.

In dem Film Ich gehe filmte er beim Besteigen eines hohen Turmes den Vorgang des Aufstiegs und nahm seine immer schnelleren Atemzüge auf (Abb. 1). Der Film dauert 2,57 Minuten. Dies entspricht der tatsächlichen Zeitspanne, die Robakowski zum Besteigen des Turms benötigte. Der Film besteht aus einer einzigen Einstellung, Aufnahme- und Vorführlänge sind identisch. Zuerst sagt der Künstler mit Bestimmtheit: „Ich gehe“, und dann zählt er laut die Stufen mit: von eins bis zweihundert. Atemnot und zunehmende Erschöpfung lassen hier das Medium Film mit der Leiblichkeit seines Schöpfers verschmelzen. Die Kamera zeigt die aufeinanderfolgenden Metallstufen, ohne dass wir auch nur einen Augenblick den Körper des Hinaufsteigenden zu Gesicht bekämen, welcher die Kamera fern des Auges in Händen hält. Der Künstler gerät immer mehr außer Atem und spricht zunehmend angestrengt die Zahlen aus. Der Atem kann, wie auch der zur Kultur- und Bildgeschichte des Pneumas forschende Kunsthistoriker Vlad Ionescu festgestellt hat, die zeitliche Natur des Bildes und Mediums denotieren. ${ }^{14}$ In Ich gehe wird dies im Prozess des Zählens ebenso wie in den Veränderungen der Atmung plastisch: Am Fuße des Turmes, wo der Aufstieg beginnt, ist Robakowskis Stimme kräftig, sein Atem kaum zu hören. Nach und nach drängen sich jedoch die beschleunigten Atemzüge in den Vordergrund, die Aussprache wird immer undeutlicher. Der Künstler keucht. Als er endlich auf der Spitze des Turmes steht, ist sein Ausatmen zu hören, so als verschnaufe er nach einer anstrengenden Tätigkeit.

Während des Aufstiegs nimmt die Kamera alle Biegungen auf, die der Künstler zurücklegt und entdeckt dabei eine für das menschliche Auge unsichtbare Welt. Die Kameraführung und die Symbiose zwischen ihr und dem Rhythmus des zunehmend erschöpften Leibes flößen beim Zuschauen Schwindel und Höhenangst ein. Die immer schnelleren Atemzüge lassen sich in diesem Zusammenhang auch als Signal nahender Gefahr lesen.

Weshalb und wozu also besteigt Robakowski den Turm? Ich würde - entgegen der Intention des Künstlers - behaupten, dass selbst solch ein Film sich durch Narrativität auszeichnet: Er spielt sich in der Zeit 

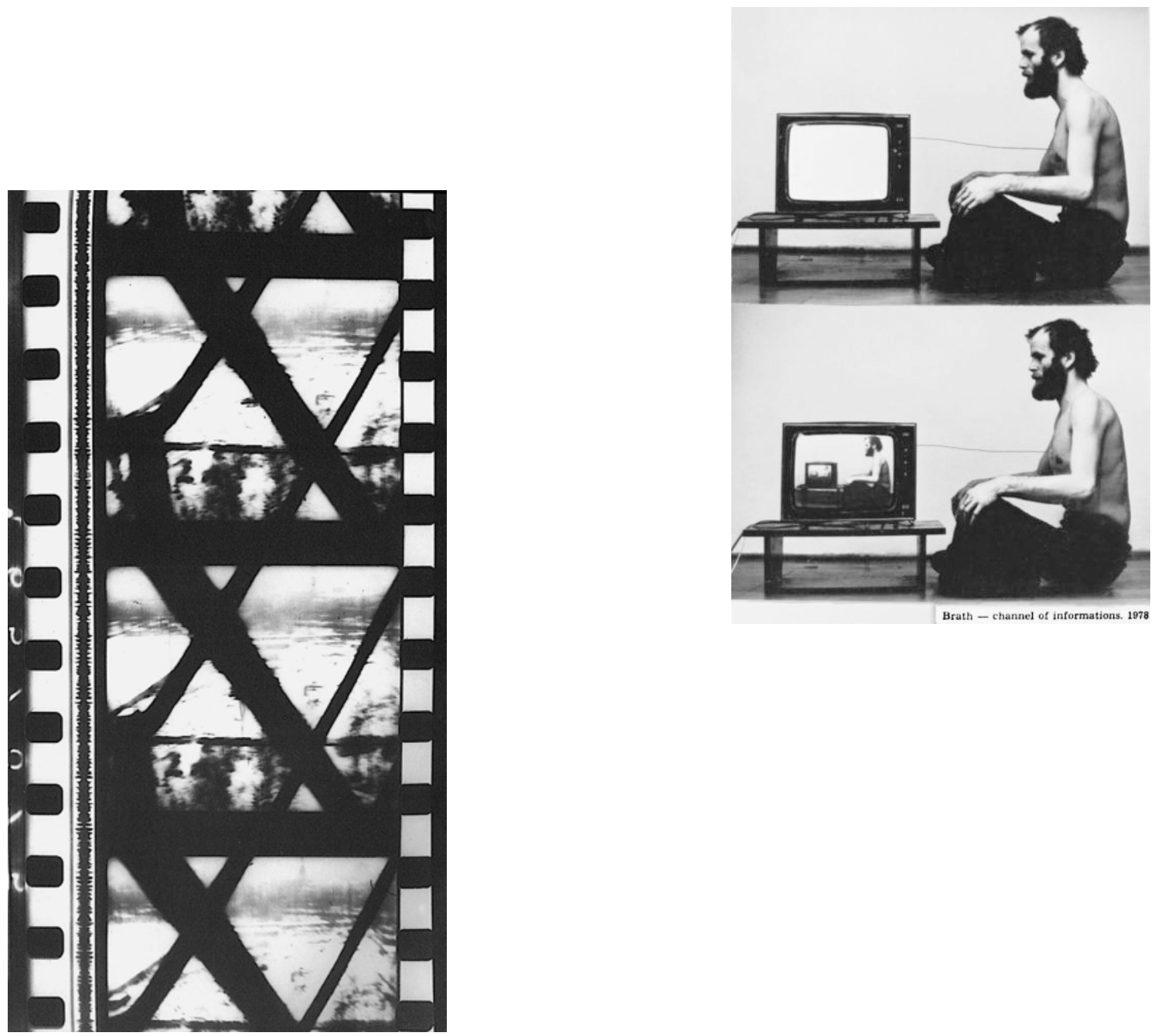

Abb. 1: Józef Robakowski, Ich gehe (Idę), Kontaktbogen der Videoperformance, 00:02:57, 1973 

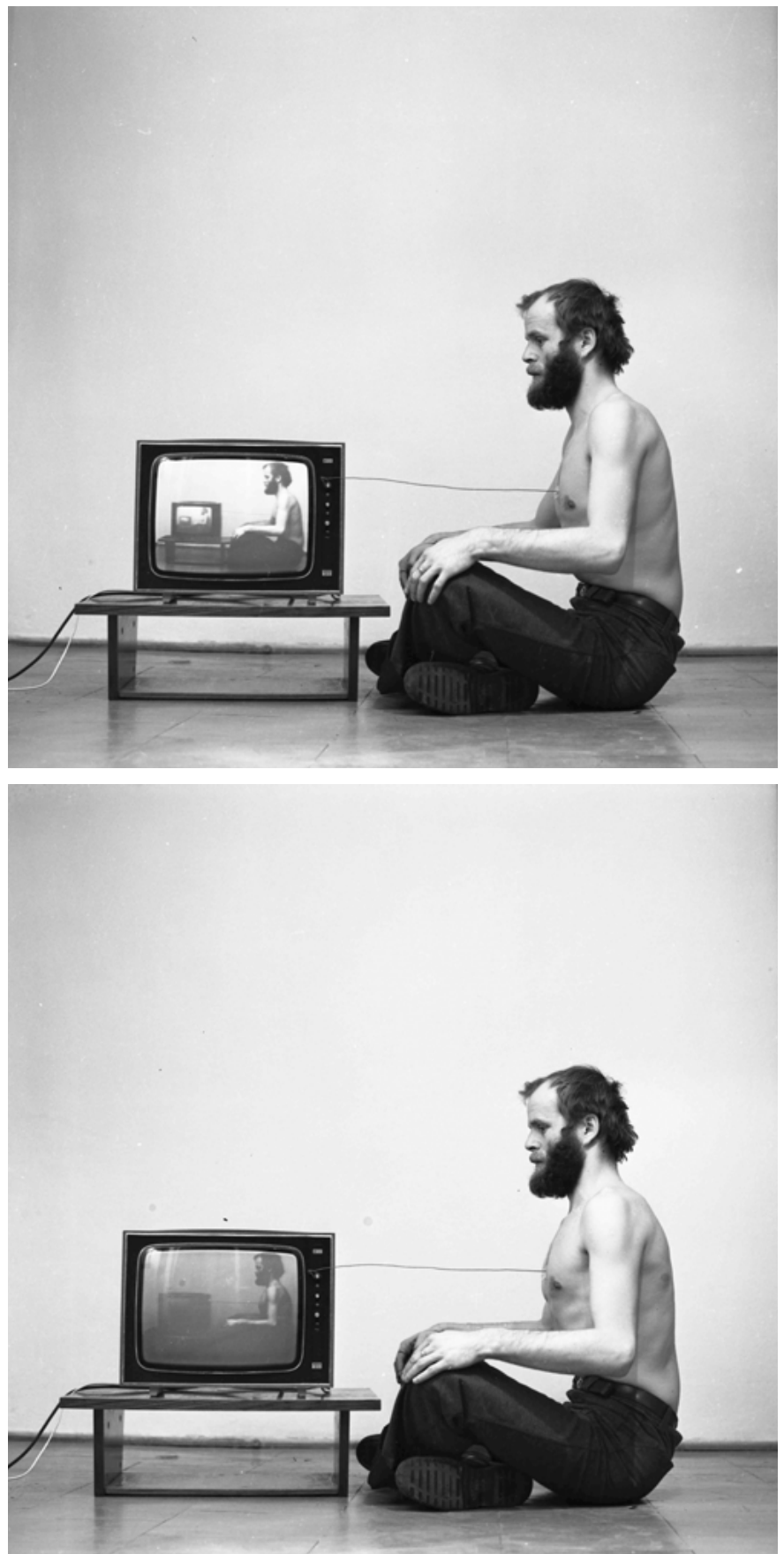

Abb. 2-4: Paweł Kwiek, Video-Atem - der Informationskanal (Video-oddech - kanał informacyjny), Screenshots, Videoperformance, 1978 
ab und erzählt die Geschichte des Aufstiegs auf einen Beobachtungsturm. Zudem erfasst die Kamera dabei die Umgebung des Turmes: die Winterlandschaft, die aus immer neuem Blickwinkel und von immer höheren Stufen sowie der Plattform aus erscheint. Wir sehen einen Wald oder Park, die Umzäunung und die Fläche eines Stadions mit Zuschauertribüne. Selbst der Beobachtungsturm bleibt nicht neutral: er bildet den höchsten Punkt, von dem aus das Gelände beobachtet, also de facto überwacht werden kann. Immer atemloser steigt Robakowski mit der Kamera hinauf. Im Kontext des Beobachtungsturmes verliert auch die Kamera ihre ohnehin zweifelhafte Neutralität, nimmt sie doch eine privilegierte Stellung ein, aus der sie von oben herab zu beobachten vermag. Sprechend ist ebenfalls, dass die Landschaft menschenleer ist und es eigentlich nichts zu sehen gibt. Die Aufmerksamkeit konzentriert sich daher umso mehr auf den atmenden Künstler.

Was ich als subversiv bezeichnen möchte, ist, dass der Künstler die Kamera mit seiner eigenen Leiblichkeit und dem Rhythmus seines eigenen Atems verknüpft. Die Kameraaufnahme repräsentiert hier also nicht die Logik der Staatsmacht und deren Öffentlichkeitsarbeit, sondern erstellt eine Form, die von Robakowski selbst „mechanisch-biologische Aufzeichnung" genannt wurde. Diese Aufzeichnung entsteht in enger Beziehung mit einem konkreten menschlichen Individuum. Es sind Robakowskis privater Atem, seine private Atemnot. Der Künstler hat noch dem trivialsten Moment der Existenz zu seinem Wert verholfen, indem er Atemzüge aufnahm und darauf hinwies, wie wichtig die alltägliche - aus der offiziellen, pathetischen Ikonosphäre des sozialistischen Polens verdrängte - Banalität der körperlichen Existenz ist. Durften doch die männlichen Helden und Erbauer des Sozialismus damals keine Atemnot haben! $!^{15}$ Es handelt sich um das Manifest eines atemlosen Antivoyeurs, ${ }^{16}$ der sich in seinem Experimentalfilm Ich gehe in seine eigene Leiblichkeit versenkt und die Funktion des Beobachtungsturmes dekonstruiert. Der Atem gilt hier also als eine würdige und gegen die Biopolitik des Staates gewandte Aktivität. Der Staat verliert die Kontrolle über den Körper des Künstlers. Sein Keuchen ist Zeichen dafür, dass er der sozialistischen Biomacht nicht mehr gehorcht. Die Machtverhältnisse legen, mit Foucault gesprochen, ihre Hand nicht mehr auf ihn. ${ }^{17}$

15 Zum Thema Männlichkeit in der Volksrepublik Polen siehe Paweł Leszkowicz, Nagi męzczyzna. Akt męski w sztuce polskiej po 1945 roku, Posen 2012.

$16 \mathrm{Zu}$ Robakowskis programmatischen Antivoyeurismus siehe Lukasz Ronduda, Sztuka polska lat 70. Awangarda, Warschau 2009, S. 281.
17 „Der Körper steht [...] unmittelbar im Feld des Politischen; die Machtverhältnisse legen ihre Hand auf ihn; sie umkleiden ihn, markieren ihn, dressieren ihn, martern ihn, zwingen ihn zu Arbeiten, verpflichten ihn zu Zeremonien, verlangen von ihm Zeichen“, zit. n. Michel Foucault, Mikrophysik der Macht. Über Strafjustiz,

Psychiatrie und Medizin, Berlin 1976, S. 37. 
Der Atem als zentraler Bestandteil des künstlerischen Produktionsprozesses findet sich in ganz anderer Weise etwa auch im Werk des zeitgenössischen deutsch-dänischen Künstlers Jeppe Hein, der mit bewusstem Atmen seine Burnout-Erkrankung therapierte und den Atem als Sitz der Lebenskraft ins Zentrum seines künstlerischen Schaffens stellte: Helen Hirsch reflektiert die Atmung in der Malerei als oft unauffälligen Aspekt in der Produktion von Werken, der erst in seiner Relevanz erkannt werden muss: „Dass auch das Erschaffen von Kunst mit der Atemtechnik verwoben sein kann, erschliesst sich einem nicht auf den ersten Blick. Bei vertiefter Betrachtung der Produktion von Werken wird erst klar, dass die Atmung ein wesentlicher Bestandteil im Entstehungsprozess sein kann.." ${ }^{18}$ In dem Film Ich gehe holte Robakowski den Atem in die bewusste Ebene und machte ihn für das Medium des Films produktiv.

Bei Robakowski wird der Kameramann zum Performer. Daraus ergibt sich eine neue Konstellation von Medium, Leib und Atem. ${ }^{19}$ Ronduda stellt die These auf, dass der Künstler die Urheberschaft seines Werkes an die Maschine - die Filmkamera - abtritt, um zu vermeiden, dass sich die Aufnahme dem Kameramann unterordnet, der durch seine eigenen Vorstellungen, Sehkonventionen und -gewohnheiten determiniert wird. ${ }^{20}$ Jedoch sei es dabei in der Reihe Mechanisch-biologische Aufzeichnungen nicht zu einer passiven Unterordnung unter die Maschine gekommen, sondern zu einem Austausch, bei dem Robakowski die Kameralogik zwar übernommen, jedoch einer ,Anthropomorphisierung' unterzogen habe. ${ }^{21}$ Hinzuzufügen ist, dass der Atem in diesem Zusammenhang als grenzüberschreitende Figur ein beide Sphären die biologisch-leibliche und die mechanische - verbindendes Phänomen darstellt. Überdies war die Struktur des Films, aus einer einzigen Einstellung bestehend und vom Atem rhythmisiert, in den 70er Jahren ganz offensichtlich politisch. Schließlich wurde bewusst auf Montage verzichtet - eine Strategie, die damals mit Illusion und Propaganda verbunden war. Diese Thematik griff, wenngleich in anderer Weise, auch Robakowskis Kollege Paweł Kwiek auf.

18 Helen Hirsch, „Vorwort und Dank“, in: Jeppe Hein.

Einatmen - Innehalten - Ausatmen, Ausst.-Kat.

Kunstmuseum Thun, Berlin 2018, S. 7-10, hier: S. 7.

19 Vgl. Guzek 2017 (wie Anm. 12), S. 203.

20 Vgl. Ronduda 2004 (wie Anm. 3), S. 281.

21 Vgl. Ebd. 


\section{Paweł Kwieks meditative Atmung: \\ Selbstporträt als Regimekritik}

Kwiek (geb. 1951) - ebenfalls aktives Mitglied der Werkstatt der Filmform - steuerte in seiner Videoperformance Video-Atem - der Informationskanal (1978) durch seinen Atem die Helligkeit des Fernsehbildes, das in der Volksrepublik Polen der Manipulation und Propaganda diente (Abb. 2-4). ${ }^{22}$ Im Folgenden wird untersucht, wie Kwiek Themen der Überwachung und Kontrolle über die Zirkulation von ein- und ausgeatmeter Luft verhandelt.

Schon in seinen früheren Arbeiten spielte der Künstler subversiv mit den Mechanismen filmischer Bedeutung und den kinematografischen Konventionen, die an der Fakultät für Kamera der Staatlichen Film-, Fernseh- und Theaterhochschule gelehrt wurden. ${ }^{23}$ Spätestens 1973 zog er auch die Objektivität der Fernsehbotschaft in Zweifel, auf welche die Zuschauer*innen keinen Einfluss hatten. Kwiek experimentierte schon damals häufig im Fernsehstudio, um die Möglichkeiten der Fernsehtechnik zu testen und gleichzeitig mit Bildern vieler verschiedener Kameras multiperspektivisch arbeiten zu können. In einem der Ausstellungsräume des Lodzer Kunstmuseums präsentierte er im Jahr 1973 einen Fernsehapparat mit ausgeschalteter Antenne, was den Empfang des von der Staatsmacht überwachten Senders verunmöglichte. ${ }^{24} 1974$ führte Kwiek bei einer Fernsehsendung über das Schaffen der Werkstatt der Filmform und seines eigenen Wirkens live Regie und erteilte den einzelnen Kameraleuten somit während der Aufnahme Anweisungen. ${ }^{25}$ Auf diese Weise gab er den Zuschauer*innen deutlich zu verstehen, dass so etwas wie eine objektive Nachrichtenübermittlung nicht existiert: Stets ist da jemand, der die gesendete Botschaft steuert und diese auf bestimmte Weise in Szene setzt. Somit betrachtete der Künstler die Filmkunst als ein Instrument des perspektivisch geplanten Gesellschaftsumbaus; Fernsehsendungen sah er als modellhaftes Beispiel an, wie die Rezipient*innen in den Zustand der passiven Aufnahme von Propaganda geführt wurden. In einem Interview unterstrich Kwiek:

„Was mich am Fernsehen fasziniert hat, ist die gleichzeitige Beobachtung von Wirkung und Ursache. Das heißt, man konnte modellhafte

22 Siehe Tadeusz Dmochowski/Marek Malinowski/Piotr Niwiński (Hg.), Media w PRL, PRL w mediach. Materiały z II Ogólnopolskiej Konferencji ,Propaganda PRL-u‘ Gdańsk 18-19 listopada 2003, Gdańsk 2004. 23 Vgl. Ronduda 2004 (wie Anm. 3), S. 284.

24 Ebd.
25 Zum Beispiel in der Arbeit Situation vom Studio (1974), siehe: https://artmuseum.pl/en/filmoteka/praca/kwiekpawel-video-a-sytuacja-studia (letzter Zugriff: 23.5.2020). 
Situationen schaffen, in denen es einen sofortigen Effekt des Handelns gab, das man beobachtete, und aus dem Beobachten den nächsten Er-kenntnisgegenstand machen, mit dem man operierte. ${ }^{\text {26 }}$

Das Mitglied der Werkstatt der Filmform untersuchte also gezielt die Beziehung zwischen dem Fernsehen als dem Medium der Macht und den Zuschauer*innen, wobei er den Akzent auf „Interaktionen des eigenen Körpers mit der technologischen Umgebung “27 legte. Er selbst beschrieb jene Interaktionen als „,informatisch-energetisches“28 Phänomen und analysierte sowohl die Auswirkung der Technologie auf den Leib als auch umgekehrt die des Leibes und Verstandes auf die Technik. Sein eigener Körper verließ also die Sphäre der Privatheit, um sich mit dem Macht- und Propagandasymbol Fernsehen auseinander zu setzen.

Die Videoperformance Video-Atem - der Informationskanal passt somit bestens in das Interessenfeld des Künstlers, der eine weitere Beobachtung der Ursache-Wirkung-Beziehung anstrebt: in diesem Falle die des atmenden Leibes und des Fernsehbildes.

Historiker*innen zufolge wurde das Fernsehen in den 70er Jahren zum ersten Mal in großem Umfang zu einem erfolgreichen Propagandainstrument des Staates, was nicht nur in Auftritten von Politiker*innen, sondern ebenso in sorgfältig ausgewählten Serien zum Ausdruck kam: ${ }^{29}$

\begin{abstract}
„Die Television ist das Olivenöl gewesen, das man auf die schaumgekrönten Wellen gesellschaftlicher Leidenschaften gegossen habe, um sie zu bezähmen. Diese Meinung kennzeichnet die Rolle, die das Fernsehen in der Erfolgspropaganda spielte. Nach den Radomer Ereignissen [Arbeiterunruhen, m.s.] von 1976 wurden die Anstrengungen noch intensiviert, die Bevölkerung davon zu überzeugen, dass es im wirtschaftlichen Sektor nichts als Erfolge gebe, und dass Probleme nur vorübergehend seien und bald verschwänden. Kurios wurde es, als im Fernsehen darum gebeten wurde, in jedem Haushalt eine Glühlam pe auszuschalten, um Energie zu sparen. Spätere Messungen ergaben, dass über eine Million Glühbirnen ausgeschaltet worden waren." ${ }^{\text {"30 }}$
\end{abstract}

Bei den oben erwähnten Werftarbeiterstreiks an der polnischen Küste im Dezember 1970 war es nämlich zu einem bedeutsamen Zwischen-

26 Tomasz Samusionek, „Wywiad z Pawłem Kwiekiem“, in: Zeszyty Artystyczne 7, 1997, S. 58-60, hier: S. 60. 27 Ronduda 2004 (wie Anm. 3), S. 286.

28 Ebd., S. 286.
29 Michał Szafran, „Telewizja Polska w latach 1945-1981. Zarys historii“, 2015, verfügbar unter: http://wiekdwud ziesty.pl/telewizja-polska-w-latach-1945-81-zarys-historii (letzter Zugriff: 3.5.2020). Vgl. auch Patryk Pleskot, „Telewizja w zyciu codziennym Polaków“, in: Dmochowski/ Malinowski/Niwiński 2004 (wie Anm. 22), S. 19-36. 
fall gekommen, welcher der Staatsmacht die Notwendigkeit zur technischen Verbesserung des Mediums verdeutlichte:
„Am 20. Dezember war geplant, vor der Ansprache Edward Giereks [des neu ernannten Ersten Sekretärs der Polnischen Arbeiterpartei, m.s.] einen Bericht aus dem ruhigen Danzig zu zeigen, doch wegen Schwierigkeiten mit der Herstellung einer ausreichend schnellen Leitung herrschte eine Zeit lang eine nervöse Stimmung, wodurch Edward Giereks Ansprache eine andere Wirkung hatte als vorgesehen. Es kam zum Verdacht der Manipulation. Und da zog der Erste Sekre- tär den Schluss, dass so rasch als möglich ins Fernsehen zu investieren sei, damit solch ein Vorfall sich nicht noch einmal ereignen könne.“31

Untersuchungen hatten schon Ende der 60er Jahre ergeben, dass sich viele, auch die regimetreuen Bürger*innen der Volksrepublik Polen dessen bewusst waren, welch großen Einfluss das Fernsehen auf ihre Meinungsbildung ausübte. Auch Kwiek war sich dessen zweifelsohne bewusst.

In der Arbeit Video-Atem - der Informationskanal hat der Künstler seinen Körper und den Fernsehapparat so verbunden, dass - wie er selbst schrieb - „durch die mechanische Verknüpfung des Helligkeitsreglers mit der Bewegung des Brustkorbs die Möglichkeit entstand, die Helligkeit durch das Atmen zu regulieren". ${ }^{32}$ Kwiek, den freien Oberkörper durch einen waagerechten Draht mit dem Helligkeitsregler verbunden, saß in der Pose eines Guru rechts vom Empfangsgerät und seitlich zu den Zuschauer*innen. Ruhig und meditativ atmete er aus, indem er die einzelnen Atemzüge wie bei einem religiösen Ritual steuerte. So wurde der Atem zu einem Medium der Informationsübermittlung, und sein Rhythmus beeinflusste die Helligkeit des Fernsehbildes auf dem Bildschirm. Momentweise erhellte der atmende Leib des Künstlers jenes Bild bis hin zu einem absolut weißen Leuchten, das in den alltäglich ausgestrahlten Sendungen unbekannt war, oder er verdunkelte es bis zu vollkommener Schwärze. Das zwischen diesen beiden Polen sichtbare Bild zeigte Kwieks mehrfaches Abbild dabei, wie er eben diese Performance ausführte (sog. Closed Circuit-Technik ${ }^{33}$ ).

31 Ebd.

32 Paweł Kwiek, „Video i oddech“, in: Uczestnictwo. Poznanie. Decyzja, Ausst.-Kat. Galeria Mała w Warszawie, Warschau 1979, o. S.

33 „Closed Circuit bezeichnet technisch gesehen die unmittelbare auditive oder visuelle Rückkopplung zwischen In- und Output eines Aufnahme- und Wiedergabesystems. Diese Rückkopplung führt zu einer Signalverstärkung und generiert ein geschlossenes Feedbacksystem. Aufgrund der gleichzeitigen aber ortsunspezifischen Aufnahme- und Wiedergabemöglichkeit

\footnotetext{
elektronischer und mittlerweile digitaler Bewegtbilder kann Video als paradigmatisches Medium des Closed Circuit-Verfahrens gelten. Durch apparative Zwischenschaltungen wie Zeitverzögerung sowie Bild- und Tonbearbeitung kann die Rückkopplung zwischen In- und Output zudem live modifiziert bzw. manipuliert werden.", zit. n.: Stephanie Sarah Lauke, „Closed Circuit-Verfahren“, in: Lexikon der Filmbegriffe, verfügbar unter: https://filmlexikon.uni-kiel.de/doku.php/c:closedcircuit verfahren-7950 (letzter Zugriff: 23.5.2020).
} 
Seine Arbeit repräsentierte damit auch eine unruhige, instabile Technik: Schwankungen in der Helligkeit.

Der polnische Künstler spielte hier auf die berühmte Arbeit $T V$ Buddha (1974) von Nam June Paik an, nahm jedoch selbst die Stelle der Buddhastatue ein und brachte das Bild mit seinem Atem vorübergehend vollständig zum Verschwinden. ${ }^{34}$ Er wählte nicht nur selbst aus, was auf dem Bildschirm zu sehen war - sein Selbstporträt beim Atmen -, sondern steuerte zudem dessen Helligkeit. Sein Einfluss sowohl auf den Inhalt als auch die Form der Botschaft war entscheidend, d. h. er usurpierte symbolisch den der Propaganda vorbehaltenen Informationskanal. Damit zeigte er sich selbst als einzelnen Bürger, dessen gleichmäßiger, meditativer Atem unerwartete Macht und Bedeutung gewann.

Kwiek ersetzte folglich die allägliche Propaganda nicht mit einem eindeutig regimekritischen, oppositionellen Inhalt, sondern stellte ihr die Privatheit und Verletzlichkeit seines halbnackten Körpers entgegen. Er zeigte damit, dass das Individuum, welches bisher nur im Privaten atmen und meditieren durfte, plötzlich und unerwartet in der Öffentlichkeit zu sehen war, was nicht zur offiziellen Ikonosphäre der Volksrepublik Polen passte. Ein- und ausatmend überschritt der Künstler so die sensible Grenze zwischen Privatheit und Öffentlichkeit. Kwiek erschien als aktiver Mensch, der Einfluss auf die Wirklichkeit nahm, obwohl er nur ganz ruhig, aber konsequent atmete. Sein durch die Closed CircuitForm virtuell unendlich vervielfachtes Selbstporträt bohrt sich hier tief in das Machtinstrument Fernsehen hinein - was vielleicht paradoxerweise politisch viel gefährlicher und wirkmächtiger war als eine inhaltliche Regimekritik. Laut Gerald Raunig, der Walter Benjamins Aufsatz „Der Autor als Produzent“ einer Relektüre unterzieht, haben solche Kunstinterventionen, die sich die Produktionsmittel aneignen, hochgradig politisches, sogar revolutionäres Potenzial. ${ }^{35}$ Die Künstler*innen besäßen so die Kompetenz zur Entwicklung von mikropolitischen Organisationsformen:

34 Vgl. Guzek 2017 (wie Anm. 12), S. 210. Die Arbeit von Nam June Paik gilt als eine "Ikone" der Videokunst und steht am Anfang einer Reihe von Closed-Circuit-Installationen und Videoskulpturen von diesem US-amerikanischen Künstler: „Die wohl bekannteste Videoarbeit von Paik entstand als Notlösung: Bei seiner vierten Ausstellung in der New Yorker Galleria Bonino ist noch eine Wand leer. Kurz vor der Eröffnung fällt ihm dafür ein, eine antike Buddha-Statue, die er einmal als Kapitalanlage gekauft hat, zum Fernsehzuschauer zu machen. Schließlich kommt noch eine Videokamera hinzu, so daß der Buddha nun sich selbst auf dem Bildschirm gegenüber betrachtet. So blicken sich Vergangenheit und Gegenwart an, so begegnen sich östliche Gottheit und westliche Medien. Bei der
Ausstellung Projekt '74 in Köln setzt Paik sich selbst in die Position des kurz zuvor entstandenen TV-Buddhas: der Gegensatz von Transzendenz und Technik, den dieses Stück enthält, liegt ebenso in seiner eigenen Person“, zit. n.: Rudolf Frieling/Dieter Daniels, Medien Kunst Netz 1: Medienkunst im Überblick, 2004, verfügbar unter: http:// www.medienkunstnetz.de/werke/tv-buddha (letzter Zugriff: 23.5.2020).

35 Vgl. Gerald Raunig, „Großeltern der Interventionskunst, oder Intervention in die Form. Rewriting Walter Benjamin's ,Der Autor als Produzent“", in: Transversal Texts 12, 2000, https://transversal.at/transversal/0601/raunig/de (letzter Zugriff: 26.5.2020). 


\begin{abstract}
„Nicht in der zum Klischee verkommenen Widerständigkeit des autonomen Kunstwerks, aber auch nicht in der plumpen Tendenz des revolutionären Sujets, sondern in der Übersetzung der formalen Fähigkeiten der KünstlerInnen vom Kunstwerk auf die Organisationsformen der Gesellschaft liegt demnach die politische Bedeutung der Kunst. “" ${ }^{“ 36}$
\end{abstract}

218 was eine simultane Beobachtung von Ursache (Aufnahmesituation) und Wirkung (übertragendes Bild) gestattete. Mit der Arbeit Video-Atem - der Informationskanal bewies Kwiek überzeugend, dass das Individuum nicht nur wirkungsvoll sein kann, sondern auch, dass seine aus dem öffentlichen Raum verdrängte ,atmende' Privatheit das Potenzial hatte, die damalige Wirklichkeit zu beeinflussen und auf mikropolitische Art und Weise neu zu organisieren. Die Übertragung seines Selbstporträts war somit ebenso politisch wie eine offene Systemkritik. Welche Gesichter im Fernsehen zu sehen waren, entschied damals schließlich noch immer die politische Kaste.

Die Möglichkeit im öffentlichen Raum in Erscheinung treten zu können gibt, Hannah Arendt zufolge, dem Menschen erst sein Realitätsgefühl. Jeder Mensch habe das Bedürfnis, gesehen und gehört zu werden: „Dass etwas erscheint und von anderen genau wie von uns selbst als solches wahrgenommen werden kann, bedeutet innerhalb der Menschenwelt, dass ihm Wirklichkeit zukommt." ${ }^{37}$ Wenn wir in der Wirklichkeit auftreten, werden wir, nach Ansicht Arendts, von Zeugen und dem Publikum beobachtet, die wiederum unsere Handlungen und Worte in Narrationen umwandeln. Kwieks Strategie, sein eigenes Gesicht in der Öffentlichkeit zu zeigen, führte also auch dazu, die Realität der Welt zu konstituieren und sich ihrer zu versichern. Im Rahmen der Arbeit Video-Atem - der Informationskanal gelang es ihm, seine Privatheit „aus der Dunkelheit des Verborgenen und Geborgenen heraustreten“" zu lassen. ${ }^{38}$

Den Fernseher - Propagandafetisch und -werkzeug der Staatsmacht, das Millionen Individuen beeinflusste - eignete sich somit ein Individuum an, das sich selbst nicht nur sichtbar machte, sondern auch unerwarteter Weise die Kontrolle über das Medium errang. Und

36 Ebd. 
zwar mithilfe des Atems, einer der grundlegendsten Vitalfunktionen. Mithilfe des unsichtbaren Atems vollzog Kwiek eine wesentliche politische Geste, indem er darauf hinwies, wie leicht die Fernsehbotschaft zu manipulieren ist. Vlad Ionescu schreibt: "The fact that breath is invisible does not mean that it signifies a void. Emptiness is actually a dynamic factor that is related to vital breath, the place where all things change.“" ${ }^{39}$ Außerdem gilt: „In den frühesten jüdischen Zeugnissen und im Alten Testament war der Atem Voraussetzung des Lebens - Gott gab Atem, Hauch und damit Geist. ${ }^{\text {"40 }}$ In Bezug auf die biblische Tradition interpretiert, kann Kwieks Handeln daher verstanden werden als eine Art Anreicherung der Fernsehbotschaft durch Geistigkeit - einer weiteren verbotenen Frucht Volkspolens. Denn das Geistige wurde aus der Erfolgspropaganda des kommunistischen, auf den Überzeugungen des dialektischen Materialismus begründeten Staates konsequent verbannt. In diesem Sinne fungierte der Atem in dieser Installation als Vehikel für das Charisma des Künstlers als Individuum. So nehmen wir Kwiek durchaus ambivalent wahr: als charismatischen Manipulateur und als verletzlichen Einzelmenschen, der sich emanzipiert.

\section{Filmatem: Subversives Potenzial zwischen Kontrolle und Kontrollverlust}

Jedes der beiden gewählten Werke zeigte einen anderen Aspekt des Atems: von der subversiven Synchronisierung des Körpers mit dem Medium Film oder dem Fernsehempfangsgerät bis hin zum metaphorischen Atemholen beim Sichbefreien aus Rollenzuschreibungen als propagandaempfänglicher, entindividualisierter und seiner Privatheit beraubter Bürger eines sozialistischen Staates. Alles spielt sich somit zwischen zwei zu Zeiten des Kommunismus verdächtigten Zuständen ab: der Atemlosigkeit und der Meditation. Robakowski keuchte und hatte seinen Atem nicht mehr unter Kontrolle, während Kwiek seine Atmungsprozesse perfekt kontrollierte. Auf „mechanisch-biologische“ und sehr bewusste Art und Weise aktivierten die beiden Künstler dabei das Potenzial des Mediums Film.

Der Atem hat mithin in der polnischen Kunst der Neoavantgarde, besonders im Kunstschaffen der Mitglieder der Werkstatt der Filmform, eine mediale, existentielle, körperliche, subversive und biopoliti-

39 lonescu 2017 (wie Anm. 14), S. 23.

40 Burkhard Baltzer, „Am Fuße des Zauberbergs. ,Atem‘: Zu den Balladen Alfred Guldens und den Zeichnungen Bettina von Haarens", in: Alfred Gulden/Bettina von Haaren (Hg.), Atem. Balladen und Zeichnungen, Merzig 2010, S. 84. 
sche sowie eine analytisch-konzeptuelle Dimension. Er ist nicht nur natürlich bedingt, sondern ebenso medial, kulturell und sozialhistorisch konditioniert sowie konditionierbar. In Robakowskis und Kwieks Handeln fungiert der Atem als Bindeglied zwischen experimenteller Form und politischem Inhalt, wobei er die Grenze verwischt zwischen der Autonomie selbstreflexiver, auf die Auslotung des Potenzials ihres eigenen Mediums konzentrierter Kunst, und dem Engagement der Kunst in der politischen Realität im Verhältnis zur Obrigkeit und zu Propaganda. Über das beschleunigte und meditative Ein- und Ausatmen öffnet sich die Kunst dieser Vertreter der polnischen Neoavantgarde hin zur Wirklichkeit und schlägt Wurzeln im konkreten Leib der Künstler. Im Rhythmus der Atmung erhalten die formalen Experimente eine die Biopolitik der Staatsmacht kritisierende Dimension.

Aus dem Polnischen von Hans Gregor Njemz. 